\title{
On Cotangent Bundles Hamiltonian Tubes Theorem and Its Some Applications in Reduction Theory
}

\author{
Abdel Radi Abdel Rahman Abdel Gadir ${ }^{1}$, Ragaa Mohammed Haj Ibrahim², \\ Nedal Hassan Elbadowi Eljaneid ${ }^{3}$ \\ ${ }^{1}$ Department of Mathematics, Faculty of Education, Omdurman Islamic University, Omdurman, Sudan \\ ${ }^{2}$ Department of Mathematics, Faculty of Education, Elzaiem El Azhary University, Omdurman, Sudan \\ ${ }^{3}$ Department of Mathematics, College of Science, Tabuk University, Tabuk, Saudi Arabia
}

Email address:

needhal ahotmail.com (N. H. E. Eljaneid)

To cite this article:

Abdel Radi Abdel Rahman Abdel Gadir, Ragaa Mohammed Haj Ibrahim, Nedal Hassan Elbadowi Eljaneid. On Cotangent Bundles Hamiltonian Tubes Theorem and Its Some Applications in Reduction Theory. American Journal of Mathematical and Computer Modelling. Vol. 4, No. 2, 2019, pp. 31-35. doi: 10.11648/j.ajmcm.20190402.11

Received: January 20, 2019; Accepted: April 25, 2019; Published: June 18, 2019

\begin{abstract}
This paper aims to study the Cotangent Bundles Hamiltonian Tubes theorem and its applications in reduction theory. The mathematical analysis method used. And found some results; The theory of reduction of cotangent bundles developed playing an important role in solution of the general problem for reduction a single or bit type cotangent bundles for base manifolds, possibility study of Hamiltonian tubes when the simplistic manifolds is a cotangent bundles, in the concrete case of cotangent bundles there is a strong motivation coming from geometric mechanics and geometric quantization that makes it desirable to obtain explicit fiber local models.
\end{abstract}

Keywords: Reduction, Cotangent Bundles, Hamiltonian Tubes, Applications

\section{Introduction}

The Cotangent Bundles $T^{*} Q$ of manifold $\mathrm{d} Q$ provides the basic model of a symplectic manifold. The Cotangent Bundles $T^{*} M$ is a smooth manifold itself, whose dimension is $2 n$. The Hamiltonian is natural energy function on the cotangent bundles. The total space of a cotangent bundles naturally has the structure of a symplectic manifold. Let $M_{n}$ be $n$ - dimensional differentiable manifold of class $C^{\infty}$ and $T^{*}\left(M_{n}\right)$ the cotangent bundles over $M_{n}$. If $x^{i}$ are local coordinates in neighborhood $U$ of a point $x \in M_{n}$. Then the Cotangent Bundles $T_{p} M$ has a dual space $T_{p}^{*} M$. In case $\mathrm{M}$ is model on Euclidean space $R^{n}$ we have $T_{p} M \approx R^{n}$ and so we want to assume that $T_{p}^{*} M \approx R^{n^{*}}$. This article concerns cotangent -lifted actions of a lie group $G$ on a cotangent bundlesT $\mathrm{T}^{*} Q$. We are motivated in part by the role of such action a groups of a symplectics of Hamiltonian systems with cotangent bundles phase spaces. Time -dependent smooth Hamiltonian on $\mathrm{T}^{*} M$, the cotangent bundles of $M$. We assume that $H$ is 1-periodic in time and grows a symptotically quadratically on each fiber. Generically, the corresponding
Hamiltonian system $x^{\prime}(t)=X_{\mathrm{H}}(t, x(t))$. We can introduce the Legendre transformation we need some basic facts about the structure of the cotangent bundles $T^{*} M$ of and $n-$ dimensional differentiable manifold $M$.

\subsection{Definition of Cotangent Space}

Given any $C^{k}$-manifold $M$, of dimensionn, with $K \geq 1$, for any $p \in M$, the tangent space at $p$, denoted $T_{p}(M)$, is the space of linear derivations on $O_{m, p}^{(k)}$ that vanish on $S_{m, p}^{(k)}$. Thus, $\mathrm{T}_{\mathrm{p}}(\mathrm{M})$ can be identified with $\left(O_{m, p}^{(k)} / S_{m, p}^{(k)}\right)^{*}$ the space $O_{m, p}^{(k)} / S_{m, p}^{(k)}$ is called the cotangent space at $p$; it is isomorphic to the dual $T_{p}^{*}(M)$, of $T_{p}(\mathrm{M})$. Observe that if $x_{i}=p r_{i} \circ \varphi$, as $\left(\frac{\partial}{\partial x_{i}}\right)_{p} x_{\mathrm{j}}=\delta_{i, j}$, the images of $x_{1}, \ldots, x_{n}$ in $O_{m, p}^{(k)} / S_{m, p}^{(k)}$ are the dual of the basis $\left(\frac{\partial}{\partial x_{1}}\right)_{p}, \ldots,\left(\frac{\partial}{\partial x n}\right)_{p}$ of $T_{p}(\mathrm{M})$. Given any $C^{k}$ - function $f$, on $M$, we denote the image of $f$ in $\mathrm{T}_{\mathrm{p}}^{*}(\mathrm{M})=O_{m, p}^{(k)} / S_{m, p}^{(k)}$ by $d f_{p}$. Using the isomorphism between 
$O_{m, p}^{(k)} / S_{m, p}^{(k)}$, and $\left(O_{m, p}^{(k)} / S_{m, p}^{(k)}\right)^{* *}$ described above, $d f_{p}$ corresponds to the linear map in $\mathrm{T}_{\mathrm{p}}^{*}(\mathrm{M})$. We see that $\left(d x_{1}\right)_{p}, \ldots,\left(d x_{n}\right)_{p}$ is a basis of $\mathrm{T}_{\mathrm{p}}^{*}(\mathrm{M})$. [8]

\subsection{Formal Definition of Cotangent Space}

The Cotangent Space $\mathrm{T}_{\mathrm{p}}^{*}(\mathrm{M})$ of a manifold at $p \in M$ is defined as the dual vector space to the tangent $\operatorname{space}_{\mathrm{p}} \mathrm{M}$. A dual vector space is defined as follow: given an $n-$ dimensional vector space $V$, with basis $E_{i}, i=1,2,3, \ldots, n$, the basis $e^{j}$ of the dual space $V^{*}$ is determined by the inner product. $\left\langle E_{i}, e^{j}\right\rangle=\delta_{j}^{i}$. When we take the basis vectors $E_{i}=\frac{\partial}{\partial x_{i}}$ for $\mathrm{T}_{\mathrm{p}}^{*}(\mathrm{M})$, we write the basis vectors for $\mathrm{T}_{\mathrm{p}}^{*}(\mathrm{M})$, as the differential line elements, $e^{j}=d x_{j}$, the inner product given by $\left\langle\frac{\partial}{\partial x_{i}}, d x_{j}\right\rangle=\delta_{j}^{i}$. Now consider the vector field $V=u^{i} \frac{\partial}{\partial x_{i}}$, and the covector field $U=u_{i} d x^{j}$, under general coordinate transformations $x \rightarrow x^{\prime}(x), V$ and $U$ are invariant, $d x_{j}{ }^{\circ}=\frac{d x_{j}{ }_{j}}{d x_{i}} d x_{i}{ }^{\circ} \frac{\partial}{\partial x_{i}}=\frac{\partial x_{j}}{\partial x_{i}} \frac{\partial}{\partial x_{j}} .[10]$

\subsection{Lemma}

The differential $d: O_{m, p} \rightarrow \mathrm{T}_{\mathrm{p}}^{*}(\mathrm{M})$ is derivation; it is a linear map for real vector spaces satisfying the Leibniz rule: $d(\emptyset . \psi)=d \emptyset . \psi+\emptyset . d \psi$, where, $\emptyset . d \psi=d \psi \cdot \emptyset$, is the cotangent vector represented by: $q \leftrightarrow \emptyset(q) .(\psi(q)-\psi(p))$.

Proof:

We want to show that $d \emptyset . \psi(p)+\emptyset(p) . d \psi-d(\emptyset . \psi)$ vanishes. It is represented by:

$(\bar{\varnothing}-\emptyset(p)) \cdot \bar{\psi}+\bar{\emptyset} \cdot(\bar{\psi}-\psi(p))-\left(\bar{\varnothing} \cdot \psi-\emptyset(p) \cdot \psi(p) \in \mathrm{J}_{\mathrm{p}}\right.$, which upon collecting terms, is equal to $(\bar{\varnothing}-\emptyset(p))$. ( $\bar{\psi}-$ $\psi(p)) \in \mathrm{J}_{\mathrm{p}}^{2}$ and hence represents zero in $\mathrm{T}_{\mathrm{p}}^{*} \mathrm{M}=\mathrm{J}_{\mathrm{p}} / \mathrm{J}_{\mathrm{p}}^{2}$. In order to relate the cotangent space. [3]

\subsection{Example}

Consider a function $f: S^{1} \times S^{1} \rightarrow R^{1}$ given by $f\left(e^{i \theta}, e^{i \emptyset}\right)$ $=\left|3-e^{i \theta}-e^{i \varnothing}\right|=\sqrt{11-6 \cos \theta-6 \cos \emptyset+2 \cos (\theta-\varnothing)}$, and so expressed in the basis of the angle charts the differential is, $d f\left(e^{i \theta}, e^{i \emptyset}\right)=(3 \sin \theta-\cos \emptyset \sin \theta+$ $\sin \emptyset \cos \theta) d \theta+(3 \sin \emptyset-\cos \theta \sin \emptyset+\sin \theta \cos \emptyset) d \emptyset /$ $f\left(e^{i \theta}, e^{i \varnothing}\right) \cdot[4]$

\section{Fiber Bundles}

\subsection{Definition}

A differentiable fiber bundles is a fiber bundles $(E, X, F, \pi, \phi, G)$, for which:

a. $X$ is an $n$-dim-differentiable manifold.

$b . F$ is $m-\operatorname{dim}$-differentiable manifold.

c. $E$ is $(m+n)-\operatorname{dim}-$ differentiable manifold.

d. $\pi: E \rightarrow X$ is a $C^{\infty}$ map, of rank $n$ every where.

$e . \phi$ is a collection of diffeomorphism.

$f . G$ is a Lie group which acts differentially and effectively. ( $g: F \rightarrow F, g \in G$ is a $C^{\infty}$ map). For a $C^{\infty}$ manifold, the tangent, cotangent and the normal frame bundles are all differentiable fiber bundles. [7]

\subsection{Remark (Restriction of Fiber Bundles)}

If $(E, M, F, q)$ is a $C^{k}-F$ bundle over $M, N \subseteq M$ is an open sub setand $E_{N}:=q^{-1}(N)$, then $\left(E, M, F, q / E_{N}\right)$ is a $C^{k}$ $F$ bundle over $N$. [1]

\section{The Cotangent Bundles of Fiber Bundles}

\subsection{Definition}

The Cotangent Space to a manifold $M$ at a point $p, \mathrm{~T}_{\mathrm{p}}^{*} \mathrm{M}$. Let us now define a fiber bundles over the space $X=M$. The fiber is $\mathrm{F}=\mathrm{T}_{\mathrm{p}}^{*}(\mathrm{M}) \sim R^{n *}$, and the total space is $E=\mathrm{T}^{*}(\mathrm{M})=$ $U_{p} \in \mathrm{T}_{\mathrm{p}}^{*}(\mathrm{M})$. (It is always true that the total space is the union of the fibers above each point). This space is called the cotangent bundle of $M$. The projection $\pi: E \rightarrow X$, becomes: $\mathrm{T}^{*}(M) \rightarrow M$, defined by: $V \in \mathrm{T}^{*}(M)=p$. Next we must give a homeomorphism, $\emptyset_{\alpha}: \pi^{-1}\left(u_{\alpha}\right) \rightarrow u_{\alpha} \otimes R^{\mathrm{n}^{*}}$. This is provided by the local coordinate on $u_{\alpha}$. If $p \in u_{\alpha}$ and its coordinate $\mathrm{s}$ are $x^{\mathrm{i}}(p) \in R^{\mathrm{n}}$, then a cotangent vector $v \in$ $\mathrm{T}_{\mathrm{p}}^{*}(\mathrm{M})$ is an element of $\pi^{-1}\left(u_{\alpha}\right)$, and its can be represent as. $\mathrm{V}=a^{i}(x(p)) \frac{\partial}{\partial x_{i}}$. This procedure defined a map, $v=(p$, $a^{i}(x(p))$. Which maps $\pi^{-1}\left(u_{\alpha}\right) \rightarrow u_{\alpha} \otimes R^{\mathrm{n}^{*}}$. If we have two different coordinate systems, then we have in $u_{a} \cap u: V$ at $p \rightarrow p\left(a^{i}(p)\right)$, coordinate $x^{i} \rightarrow p\left(b^{i}(p)\right)$, coordinate $y^{i}$. [12]

\subsection{Definition}

The Cotangent Bundles $\mathrm{T}^{*}(M)$. is the space of position and moment $E=\left\{x, p ; x \in M, p \in \mathrm{T}_{\mathrm{x}}^{*}(\mathrm{M})\right\}$. This $B=M$ and $F_{x}=$ $\mathrm{T}_{\mathrm{x}}^{*}(\mathrm{M})$, with

$$
\pi=\mathrm{T}^{*}(M) \rightarrow M,(x, p) \rightarrow x
$$

A fiber bundle has more structure since the fibers $F$ must lie in side $E$ in a special way which is locally a product. We define it as a quintuple $\{E, \pi, B, F, G\}$ consisting of:

a) A manifold $E$, projection map $\pi$, basic space $B$, fiber $F$ together with a structural group $G$ of diffomeomorphism of $F$ acting on the left.

b) An atlas of charts, a covering of $B$ by open sets $u_{\mathrm{i}}$, where $\emptyset: \pi^{-1}\left(u_{i}\right) \rightarrow u_{i} \times F(2)$. Where, $\varnothing_{i}(p)=$ $\left\{\pi(p), g_{i}(p)\right\}, p \in \pi^{-1}\left(u_{i}\right)$ (3). And $g_{i}: \pi^{-1}\left(u_{i}\right)$ $\rightarrow F$. (4). Moreover, if we define the restriction.

$g_{i}(x)=g_{i} / F_{x}$. Then $g_{i}(x): F_{x} \rightarrow F$ is a left action of $G$ on F. [16]

\subsection{Definition}

A map (or morphism) of fiber bundle $\left(F_{1}, E_{1}, B_{1}\right) \rightarrow\left(F_{2}\right.$, $\left.E_{2}, B_{2}\right)$, is a pair of base point preserving continuous maps. $\varnothing: E_{1} \rightarrow E_{2}$ and $\varnothing: B_{1} \rightarrow B_{2}$ making the following. 


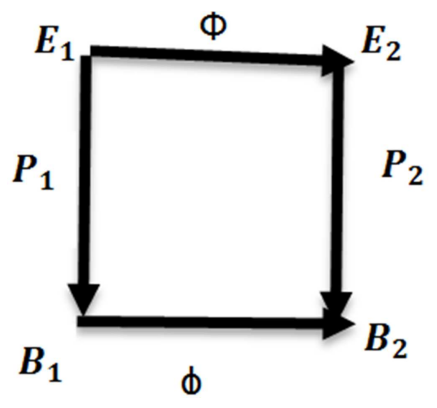

Figure 1. Map of Fibrations.

Notice that such a map of fibrations determines a continuous map of the fibers $\emptyset_{0}: F_{1} \rightarrow F_{2}$. A map of fiber bundles. $\left(F_{1}, E_{1}, B_{1}\right) \rightarrow\left(F_{2}, E_{2}, B_{2}\right)$ is an isomorphism if there

Is an inverse map of fibrations.

$\emptyset^{-1}\left(F_{2}, E_{2}, B_{2}\right) \rightarrow\left(F_{1}, E_{1}, B_{1}\right)$ so that $\emptyset_{0} \circ \emptyset^{-1}=\emptyset^{-1}$ 。 $\emptyset_{0}=1$.

Finally, we say that fibration $(F, E, B)$ is trivial if it isomorphic to the trivial fibrations $B \times F \rightarrow B$. [7]

\subsection{Some Examples}

a) The projection map $X \times F \rightarrow X$ is the trivial fibration over $X$ with fiber $F$.

b) Let $\exp : R \rightarrow S^{1}$ be given by $\exp (t)=e^{2 \pi i t} \in S^{1}$. Then exp is locally trivial fibrations with fiber the integers $Z$.

c) Recall that the $n$ - dimensional real projective space $R p^{n}$ is defined by $R p^{n}=S^{n} / N$, where $x^{\sim}-x$, for $x \in S^{n} \subset R^{n+1}$. Let

$p: S^{n} \rightarrow R p^{n}$ be the projection map.

This is a locally trivial fibrations with fiber the two points set. [9]

\subsection{Theorem}

Let $(E, B, F, p)$ be a locally trivial fiber space whose total space and base space are path -connected and $X$ a pathconnected topological space. For the mapping. $\varnothing: X \rightarrow B$ to have a lift $\Psi$ satisfying the condition $\left(x_{0}\right)=e_{0}$, where $x_{0} \in$ $X, e_{0} \in E, p\left(e_{0}\right)=b_{0}=\left(x_{0}\right)$, it is necessary that $\emptyset_{n}\left(\pi_{\mathrm{n}}\left(X, x_{0}\right)\right)$ $\subseteq p_{n}\left(\pi_{\mathrm{n}}\left(E, e_{0}\right)\right)(3.5)$. For all $n \geq 1$.

Proof:

If such a lift $\Psi$ exists, then diagram is Commutative. using factors of homology groups, we obtain the commutative diagrams (for all $n \geq 1$ )

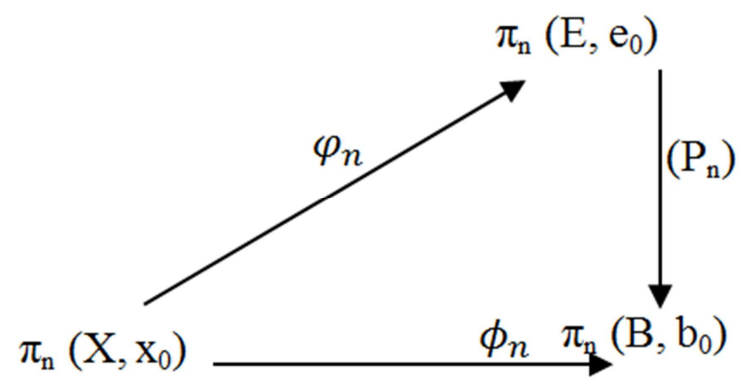

Figure 2. From which the required inclusions follow easily. [18]

\subsection{Example}

For each smooth $n$-dimensional manifold $M$, the cotangent bundles $\mathrm{T}^{*} \mathrm{M}$, is a vector bundle $\left(\mathrm{T}^{*} M, M, R^{\mathrm{n}^{*}}, q\right)$. [14]

\subsection{Definition of Principal Bundles}

Let $G$ be a lie group $k \in N \cup\{\infty\}$. A $C^{k}$-principal bundles is a quintuple $(p, M, G, q)$, where: $p \times G \rightarrow p$ is a $C^{k}$-right at action with the property of local triviality: Each point $m \in M$ has an open neighborhood $U$ for which there exists a $C^{k}$-diffeomorphism.

$\varphi_{u}: u \times G \rightarrow q^{-1}(u)$, satisfying $q \circ \varphi_{u}=p_{u}$ and the equivariance property. $\varphi_{u}(u, g, h)=\varphi_{u}(u, g) . h$, for $u \in$ $U, g, h \in G$. [6]

\subsection{Example}

The Cotangent Bundles $\mathrm{T}^{*} Q$ contains the following classes of Lagrangian submanifolds; The fibers of $\mathrm{T}^{*} Q$. Let $x \in Q$ and let $i ; \mathrm{T}_{\mathrm{x}}^{*} \mathrm{Q} \rightarrow \mathrm{T}^{*} Q$ be the natural inclusion mapping. Since $T_{x}^{*} Q$ has half the dimension of $\mathrm{T}^{*} Q$, it is enough to show isotropy. For $\xi \in \mathrm{T}^{*} Q$ and $X \in T_{\xi}\left(\mathrm{T}_{\mathrm{x}}^{*} \mathrm{Q}\right)$, we find $\left(i^{*} \theta\right)_{\xi}(X)=$ $\theta_{i}(\xi)(i X)=\langle i(\xi), \pi \circ i(x)\rangle=0$. Thus $i^{*} \theta=0$. In bundles coordinates the proof is even simpler: Since $q^{\mathrm{i}}$ is constant along $T_{x}^{*} Q$, we obtain $i^{*}\left(d p_{i} \cap d q^{i}\right)=0$. $[7,11]$

\section{Hamiltonian Dynamics on Cotangent Bundles}

\subsection{Definition}

Let $M$ be the configuration space of a classical system with regular Lagrangian $L, T M$ the velocity phase space and $T^{*} M$ the momentum phase space. The Legendre transformation. $A: T M \rightarrow T^{*} M$, to make the transition from the Lagrangian to the Hamiltonian formula $M_{x}$. The Lagrange equation can be characterized invariantly on $T M$ as the differential equations for the integral curves of the vector field $X_{\varepsilon}$ defined by $\left.d h=-x_{\varepsilon}\right\lrcorner \omega_{\mathrm{c}}$, where $\omega_{\mathrm{c}}=A^{*}(\omega)$, and $h \varepsilon=A c-\varepsilon=\varepsilon(\varepsilon-c)$. Our goal now is to reformulate these equations on $\mathrm{T}^{*} M \cdot$. [15]

\subsection{Definition}

Two fundamental aspects of Hamiltonian dynamics that is central to both the canonical quantization scheme and geometric quantization are the Poisson bracket and the related Lie algebraic structure of $C^{\infty}$ - function on $T^{*} M$. Let $M$ denoted a differential form and $X$ a smooth vector field on $T^{*} M$. From differential geometry we have following general formula that relates the exterior derivative operator $\mathrm{d}$, the Lie derivative operator $L X$, and the (left) hook product \lrcorner$: L X(\mu)=X\rfloor d(\mu)+d(X\rfloor \mu)(5)[6]$

\subsection{Definition}

The set of all locally Hamiltonian vector fields on $T^{*} M$ is denoted by, $L H V \equiv L H V\left(T^{*} M\right)$, and the set of Hamiltonian vector fields on $T^{*} M$ is denoted by: $H V \equiv H V\left(T^{*} M\right)$ [13] 


\section{Splitting of Cotangent Bundles}

\subsection{Definition}

Let $U \subset B$ be the nilpotent radical of $B$. Let $B \subset p$ b a (standard) parabolic subgroup with the nilpotent radical $U_{p}$ and the component $L_{p}$ containing $T$; so opposite parabolic $p$. We denote the Lie algebras of $G, p, B, T, U, U_{p}, \mathrm{~L}_{\mathrm{p}}$, by the corresponding Gothic characters $\mathrm{g}, p, b, t, u, u_{p}, l_{p}$, respectively. By a volume form on a smooth variety $X$, we mean now here vanishing differential form top degree on $X$. [5].

\subsection{Definition}

A differential 1-from $\theta \in \Omega^{1}(N)$ is called an exact 1-form if there exists a smooth function $g: N \rightarrow R$, such that $\theta=d g$. The notation for cotangent vectors. Let $N=R$, and $\theta$ a 1 form on $N$ given in local coordinates by $\theta_{t}=h(t) d t$, which can be identified with a function $h$. The notation makes sense because $\theta$ can be integrated over any interval $[a, b] \subset$ $R: \int \theta[a, b]:=\int_{a}^{b} h(t) d t$. Let $M=R$, and consider a mapping $f: M=R \rightarrow N=R$, which satisfies $f^{\prime}(t)>0$. Then $t=f(s)$ is an a appropriate change of variables. Let $[c, d]=f([a, b])$, then:

$$
\int f^{*} \theta=\int_{c}^{d} h(f(s)) f^{\prime}(s) d s=\int_{a}^{b} h(t) d t=\int \theta[\mathrm{c}, \mathrm{d}]
$$

which is the change of variables formula for integrals. [7]

\subsection{Example}

If $f(x, y)=x^{2} y \cos x$ on $I R^{2}$, then $d f$ is given by the formula $d f=\frac{\partial\left(x^{2} y \cos x\right)}{\partial x} d x+\frac{\partial\left(x^{2} y \cos x\right)}{\partial y} d y$ $=\left(2 x y \cos x-x^{2} y \sin x\right) d x+x^{2} \cos x d y$. [2]

\subsection{Slice Theorem}

Let $H$ be a Lie subgroup of a Iie group $G$, and $S$ a manifold on which $H$ acts. Consider the following two left actions on $G \times S$ :

The twist action of $H: h .(g, s)=\left(g h^{-1}, h . s\right)$, the left multiplication of $G: g^{\prime} .(g, s)=\left(g^{\prime} g, s\right)$. these are easily seen to be free and proper. The twisted product $G \times H^{S}$ is the quotient of $G \times S$ by the twist. It is a smooth manifold; in fact $G \times H^{S} \rightarrow G / H$ is the fiber bundles associated with the principal bundles $G \rightarrow G / H$ via the $\mathrm{H}$ on $\mathrm{S}$. The left multiplication commutes with the twist and descends to a smooth on $\mathrm{G} \times H^{s}$, namely $g^{`} .[g, s] H=[g, g, s] H$. Now consider a $G$ on a manifold. Let $z \in M$, with isotropy subgroup $H=G_{z}$. A tube for the $G$ action at $z$ is a $G$-equivariant diffeomorphism from some twisted product $G \times H^{s}$ to onopen neighborhood of $\operatorname{zin} M$, that maps $[e, 0]_{\mathrm{H}}$ to $z$. The space $N$ may be embedded in $G \times H^{s}$ as $\{[e, s] H: s \in S\}$; the image of the latter by the tube is called a slice theorem. A slice theorem (or tube theorem) is a theorem guaranteeing the existence of a tube under certain conditions. [12].

\section{A Cotangent Bundles Hamiltonian Tubes Theorem and Its Applications in Reduction Theory}

In this part we study the symplectic geometry of cotangent -lifted action induced by a smooth proper action of a Lie group on a smooth manifold. Symplectic manifolds have their origin in the geometric for Hamilton's and Lagrange's equations of classical mechanics, where symmetries is the main tool that can be used to simplify the equations of motion. This model is known as the Hamiltonian tubes; it the basis of almost all the local studies concerning Hamiltonian of Lie groups on symplectic manifolds. It is applications has been limited by the fact the proof is no constructive. In the first part of thesis we are going to study Hamiltonian tubes when the symplectic manifolds is a cotangent bundles. In the concrete case of cotangent bundles there is a strong motivation coming from geometric mechanics and geometric quantization that makes it desirable to obtain explicit fiber local models. The first work studying symplectic normal forms in the specific case of cotangent bundles.

\subsection{Regular Cotangent Bundles Reduction}

The symplectic reduction of the cotangent bundles $T^{*} \mathrm{Q}$ has more structure than a symplectic manifold. In this we recall the results that characterize the reduced space as a subset of a certain cotangent bundles. [17]

\subsection{Theorem (Regular "Point" Cotangent Bundles Reduction at Zero)}

Let $G$ act freely and properly by cotangent lifts on $T^{*} \mathrm{Q}$, and let Jbe the momentum map of the $G$ action (with respect to the canonical symplectic form on $T^{*} Q$. Let $\pi_{G}: Q \rightarrow Q / G$ is projection. Define the map, $\emptyset: J^{-1}(0) \rightarrow T^{*}(Q / G)$ by, for every $p \in T_{q}^{*} \mathrm{Q}$. Then $\emptyset$ is $a G$, invariant surjective submersion and descends to a symplectic homeomorphic. The left -hand side has the reduced symplectic form corresponding to the canonical symplectic form on $\mathrm{T}^{*} \mathrm{Q}$, and $\mathrm{T}^{*}(\mathrm{Q} / \mathrm{G})$ has the canonical symplectic form. The map $\emptyset$ is a sort of pushforward, though $\pi_{G}$ is not injective. Note that $\emptyset$ is "injective $\bmod G "$, meaning that $\emptyset\left(z_{1}\right)=\varnothing\left(z_{2}\right)$ if and only if $z_{1}=g \cdot z_{2}$ for some $g \in G$. [12]

\section{Results}

The first result of the theory in cotangent bundles reduction, the theory developed for the problem with a single or bit type playing an important role in the solution to the general problem of a singular cotangent bundles reduction for base manifolds, Hamiltonian tubes when the symplectic manifolds is a cotangent bundles, in the concrete case of cotangent bundles there is a strong motivation coming from geometric mechanics and geometric quantization that makes it desirable to obtain explicit fiber local models and the first work studying symplectic normal forms in the specific case of cotangent bundles. 


\section{Conclusion}

Conclude that the theory of reduction of cotangent bundles developed playing an important role in solution of the general problem for reduction a single or bit type cotangent bundles for base manifolds and found that the phase space is the cotangent bundles $\mathrm{T}^{*} \mathrm{Q}$ of a configuration space $Q$.

\section{References}

[1] A. A. Kirillov Lectures on the Orbit Method; volume 64, of Graduate studies in mathematics, springer. American mathematical society, printed in the USA, 2004.

[2] Bernard. F. Schulz, geometrical method of mathematical physics, volume 296, Cambridge university press, 1980

[3] G. J. Rudderpost, introduction to differentiable manifolds, second edition, Springer, New York Verlag, 2012.

[4] Hyman Bass Joseph, differential geometry and analysis on manifolds, volume 246, Springer, United States of America, 2006.

[5] Brian. Kumar, frobenius splitting methods in geometry and representation theory, part III, progress in mathematical, 2005.

[6] G. W. Gibbons, application of differential geometry to physics, part III, Wilber Forc Road, UK, 2006.

[7] James J stoker, differential geometry, wily and sons New York, London, 1969.

[8] Jeffery. M. Lee, differential geometry, analysis and physics, 2000 .
[9] John M. Lee. Introduction to smoothmanifolds, volume 218 of Graduate Texts in mathematics, Springer, New York, second edition, 2013.

[10] Joel. W. Robbin and Ditmar Asalamon, introduction to differential geometry, third edition, university of Wisconsin, Madison, 2013.

[11] John Opera, differential geometry and its applications, second edition, mathematical association of America eleveland state university, 2012.

[12] Larry K Norris, symplectic geometry on tangent and cotangent bundle, North coralina state university, 2003.

[13] M. crampin, lifting geometric object to a cotangent bundles and geometry of cotangent bundles of a tangent bundles, Walton Hall, krijgslaan, 281, Belgium.

[14] Marian Fecko, differential geometry and lie group for physicists, third part Comenius university. Bratislava Slovakia, Cambridge, 2006.

[15] $\mathrm{Ph} . \mathrm{d}$. thesis, cotangent bundle Hamiltonian tube theorem and its applications in reduction theory, faculty matermatiques Estaristica university polite cicada catal, 2010.

[16] S. Akbulut. M. Ozdemir A. A. Salimov, diagonal lift in the cotangent bundles and it application Turk J. mathtubi tak, 2001.

[17] Simonhochgerner, Singular Cotangent Bundles Reduction and spin cologero- Moser systems Wien - Vienna, 2005.

[18] Sunil Mukhi N. Mukunda, introduction to topology, differential geometry and group theory for physicists, copyright. Wiley Eastern Limited, 1990. 